\title{
The Effects of Head Support on Muscle Activity and Pain in a Forward-leaning Posture
}

\author{
Kang-hee Kim, BS, Yoon-hee Ko, BS, Tae-lim Yoon, PT, PhD \\ Department of Physical Therapy, College of Health and Medical Science, Cheongju University, Cheongju, Korea
}

\author{
Article Info \\ Received October 7, 2020 \\ Revised October 31, 2020 \\ Accepted November 2, 2020 \\ Corresponding Author \\ Tae-lim Yoon \\ E-mail: taelimyoon@cju.ac.kr \\ https://orcid.org/0000-0002-1718-2205
}

\section{Key Words}

Electromyography

MyoVIDEO

Orthotic devices
Background: Because a forward-leaning posture can cause increased back muscle activity and pain. Therefore, an innovative method to reduce back muscle activity and pain is required.

Objects: This study aimed to investigate the effects of a head support on muscle activity and pain in a forward-leaning posture.

Methods: A total of 14 male and 16 female students (average age, $21.65 \pm 2.37$ years; height, $166.15 \pm 7.90 \mathrm{~cm}$; and weight, $60.65 \pm 9.00 \mathrm{~kg}$ ) were recruited for the experiment. Two of them were excluded due to musculoskeletal disorders. The muscle activity and pain in the forward-leaning posture were assessed while participants washed dishes for 7 minutes with and without a head support. The condition of using a head support was randomly performed with a 5-minutes break. To confirm a lumbar flexion angle of $30^{\circ}$ during the experiment, myoVIDEO was used, and surface electromyography was used to measure muscle activity. Pain was assessed using a 10-point visual analog scale (VAS). The Wilcoxon signed-rank test was used to analyze the data, with $p<0.05$ indicating statistical significance.

Results: The cervical, thoracic, and lumbar erector spinae muscle activities significantly decreased with the use of the head support, but there was no significant change in the gluteus maximus. There was a significant decrease in the VAS score for the lumbar erector spinae $(p<$ 0.05), but there was no significant change in the VAS score for the cervical region.

Conclusion: The use of a head support in a forward-leaning posture reduced cervical, thoracic, and lumbar erector muscle activity and pain. Therefore, it could be recommended during working in a forward-leaning posture, such as during dishwashing, cooking, and working as a factory employee.

\section{INTRODUCTION}

When standing and working for an extended duration, a forward-leaning posture can cause musculoskeletal disorders. A forward-leaning posture causes the center of the body mass to move forward and causes excessive contractions of the back muscles to maintain the posture [1]. Prolonged standing at work and a forward-leaning posture have been associated with serious health consequences, such as leg or back pain, cardiovascular problems, fatigue, and discomfort, and approximately $50 \%$ of healthy participants report low back pain (LBP) after two hours of standing long-term [2,3]. People who use large, deep sinks, such as chefs, bend forward for long durations when preparing food or washing dishes, which provides a heavy workload on the low back, which is a risk fac- tor for back pain [4]. Repetitive bending or inconsistent body postures, which increase the amount of work of the waist and legs, are also major risk factors for the development and exacerbation of LBP [5].

To prevent LBP that can occur while working in a forwardleaning posture, a short break to relieve muscle fatigue or height adjustment of the work surface or kitchen counter have been used to reduce back muscle activity and discomfort [6,7]. One study prevented LBP by adjusting the height of the legs of the work surface, but this strategy was disadvantageous when several people worked and had a limitation in the adjustment of the worktable or care taken for long-term use [6]. In another study, a kitchen counter was used to relieve the activity of the lumbar erector spinae in the forward-leaning posture [7]. This strategy reduced discomfort in the low back and low back 
muscles [8]. When using the kitchen counter, the muscle load on the low back and leg muscles decreases, but neck and back flexion are increased, which can cause back pain and subjective discomfort [9].

Using a head support to reduce the mass of the body in a forward-leaning posture reduces muscle activity and pain in the cervical and lumbar regions. A previous study reported that supporting the body using a chest support, which was designed for surgeons who often lean forward over the surgical field, was effective in reducing muscle activity (40\%) in the low back muscles in a forward-leaning posture. The reduced low back muscle activity also minimized LBP $[10,11]$. However, the chest support disrupted participants' breathing because of the pressure on the chest and only affected the lumbar region. Using a head support with a different design could solve these problems.

The purpose of this study was to evaluate the effects of a head support on cervical, thoracic, and lumbar erector muscle activity and pain in the cervical and lumbar regions in a forward-leaning posture. The hypothesis of this study was that there are significant changes in the activity of muscles and pain in the cervical, thoracic, and lumbar erector spinae with the use of a head support.

\section{MATERIALS AND METHODS}

\section{Participants}

The participants were male and female students who were recruited with a recruitment notice from September 2019 to November 2019 at a University. Before the experiment, all participants received a full explanation of how they would be measured and provided written informed consent. The selection criteria were as follows: 1) Those who had not had back pain in the last 6 months; 2) those who did not have a history of lumbar or other musculoskeletal disorders [12,13]; 3) those who did not have psychiatric problems; and 4) those who were not pregnant. A total of 32 students were recruited at the beginning of the study, two of whom were excluded for a diagnosis of lumbar intervertebral disc escape. The participants' characteristics are shown in Table 1.

\section{Equipment}

\section{1) Head support}

A head support was used to distribute the weight of the body along the support during dishwashing (Figure 1A). Figure $1 \mathrm{~B}$ shows the details of the model for bending forward while leaning against a chest support. The upper body weight $(\mathrm{Fg})$, the back muscle force (Fm) at the level of L5 (lumbar), and the supporting force (F support) are included in this biomechani-

Table 1. The descriptive characteristic of the participant

\begin{tabular}{lrrr}
\hline \multicolumn{1}{c}{ Variable } & Male $(\mathrm{n}=14)$ & Female $(\mathrm{n}=16)$ & Total $(\mathrm{N}=30)$ \\
\hline Age $(\mathrm{y})$ & $22.00 \pm 3.37$ & $21.30 \pm 0.87$ & $21.65 \pm 2.37$ \\
Height $(\mathrm{cm})$ & $172.70 \pm 5.67$ & $159.60 \pm 2.75$ & $166.15 \pm 7.90$ \\
Weight $(\mathrm{kg})$ & $66.10 \pm 8.40$ & $55.20 \pm 6.04$ & $60.65 \pm 9.00$ \\
Body mass index $\left(\mathrm{kg} / \mathrm{m}^{2}\right)$ & $22.07 \pm 2.05$ & $21.70 \pm 2.46$ & $21.83 \pm 2.25$ \\
\hline
\end{tabular}

Values are presented as mean \pm standard deviation.
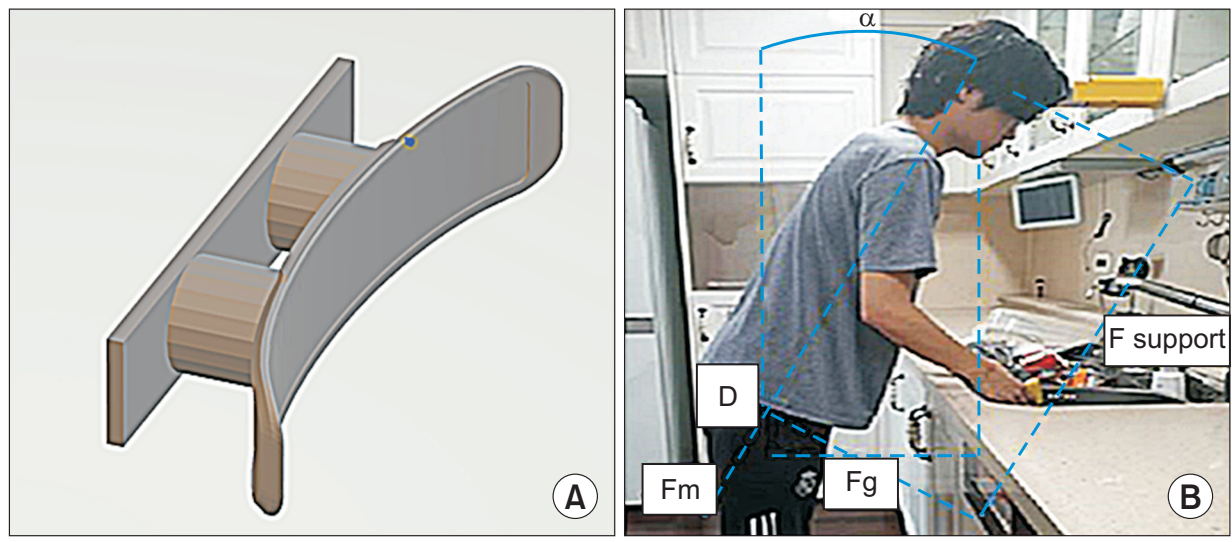

Figure 1. Applying of head support (A, model of head support; $B$, model of forward leaning posture and reaction forces). $\mathrm{Fg}$, upper body weight; Fm, back muscle force; F support, supporting force; D, the equilibrium of moment of forces in the sagittal plane at position $D$. 
cal model. Note that the model is limited to the sagittal plane and describes a static equilibrium. The mass center of gravity of the upper body is located near the axillae. Considering the equilibrium of the moment of forces in the sagittal plane at position $\mathrm{D}$, the lower Fm can be calculated as follows:

Without Support $(\mathrm{F}$ support $=0) \mathrm{Fm}=\mathrm{Fg}$ Equation (1) With Support $($ F support $=0) \mathrm{Fm}=\mathrm{Fg}-\mathrm{F}$ support Equation (2)

According to Equation (2), a head support might be most effective in reducing the Fm in the low back, since distance $\mathrm{c}$ is at a maximum (and thus F support is maximized) [14]. Based on this information, the head restraint can achieve more than $65 \%$ efficiency. The head support is installed perpendicular to the wall and is designed to adjust the height according to the height of the participant. The participants were ordered to bend $30^{\circ}$ from an upright posture and stand with their foreheads against the head support. If the head support fell or the participants' posture collapsed during dishwashing, the measurements were stopped and remeasured.

\section{2) MyoVIDEO}

The Noraxon MyoVIDEO system was used with a NiNox 125/250 camera (Noraxon USA Inc., Scottsdale, AZ, USA) to record and monitor the lumbar flexion angle during the dishwashing motion $[15,16]$. MyoVIDEO was used to continuously collect displacement data from all four markers at the upper part of the sacrum, greater trochanter of the femur, spinous process of C7, and lower part of the ear [17]. The sensors were attached to the skin using tape. The angular direction was normalized to a standing upright position, which was recorded before each test. Therefore, the participants were instructed to maintain lumbar flexion to $30^{\circ}$ during the task in a forwardleaning posture. If there was an angle change of more than \pm $10^{\circ}$ during the task in the forward-leaning posture, the measurement was stopped and remeasured the following day.

\section{3) Surface EMG}

In this study, surface electromyography (sEMG) was used to measure the muscle activity of the erector spinae (cervical, lumbar, and thoracic) and the gluteus maximus. A Noraxon DTS (Noraxon Inc., Scottsdale, AZ, USA) was used to collect sEMG data. To measure muscle activity, the electrodes were attached while maintaining the distance between the electrodes
$2 \mathrm{~cm}$ perpendicular to the muscle fiber. An sEMG attachment site was attached to $2 \mathrm{~cm}$ of C4, T12, L1, L5, the great trochanter, and S2 to one-third of the crypt line (Figure 2). To minimize skin resistance, all areas were cleaned with alcohol and completely dried before electrode attachment. The following parameters were set: sampling rate, 1,500 Hz; notch filter, $60 \mathrm{~Hz}$; and band-pass filter, 20-500 Hz. All sEMG data were calculated using Noraxon MR 3.8 software [18]. In addition, maximum voluntary isometric contraction (MVIC) was used to determine the normalized value of muscle activity. When measuring the MVIC of each muscle, the sEMG of the maximum effort state was repeatedly measured three times for 5 seconds each. The value for 3 seconds in the middle except for the first and the last of the root mean square values measured for 5 seconds was selected, and the average value of the repeated EMG over 3 seconds was obtained and used as a normalized value (\%MVIC).

\section{4) Visual analogue scale}

Subjective ratings of cervical and low back pain were collected at the completion of the task in each condition. Low back and cervical pain were assessed using a fixed 10-point visual analogue scale (VAS) from no pain to extreme pain [19].

\section{Procedures}

Initially, the test participants practiced through a familiarization process for 5 minutes. Participants performed the dishwashing motion for 7 minutes with the head support and

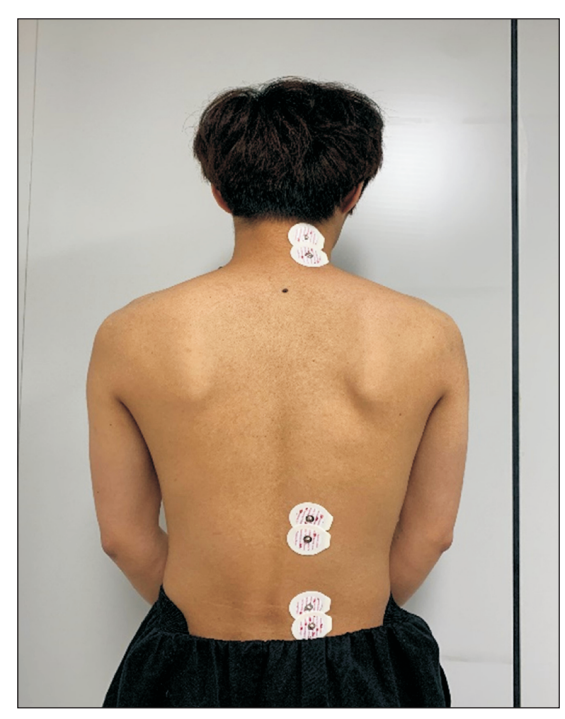

Figure 2. Placement of surface electromyography electrode. 
7 minutes without head support. The sequence for trials was randomized (Figure 3) [20]. During the test, the participants' lumbar angles were maintained at $30^{\circ}$. Verbal cues were provided to maintain the angle while the researcher continuously observed the body posture. If a change of more than $\pm 10^{\circ}$ oc$^{-}$ curred for more than 5 seconds, the experiment was stopped and resumed the following day. The participants did not lean on the sink with their waists, and their feet were placed 10 $\mathrm{cm}$ away from the bottom of the sink. They were instructed to stand with their feet apart at pelvic width. The participants were instructed to do the dishes without washing any special sections. The dishes were washed at a speed of 20 bowls per minute. Tape markings on the floor were used to maintain a consistent foot position and posterior distance from the sink throughout testing (Figure 4A). The head was supported by the head support (Figure $4 \mathrm{~B}$ and $\mathrm{C}$ ). The remaining tasks were performed with the same procedure as above. Tasks with and without the head support were conducted in random order, and 5 min of rest was provided between the tasks [21]. In addition, EMG was simultaneously measured. After the two tests,

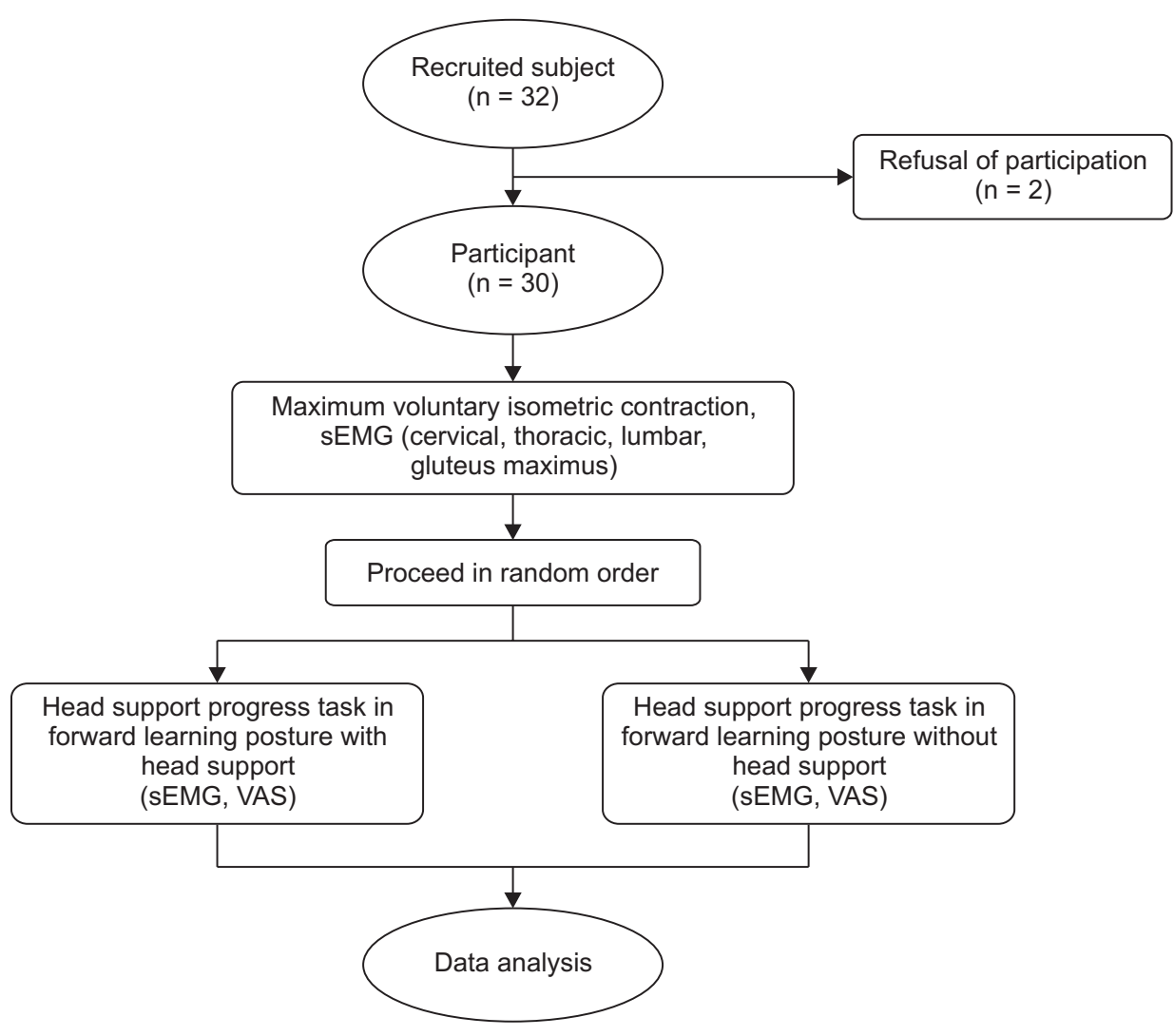

Figure 3. Fowchart: procedure with and without head support. sEMG, surface electromyography; VAS, visual analog scale.

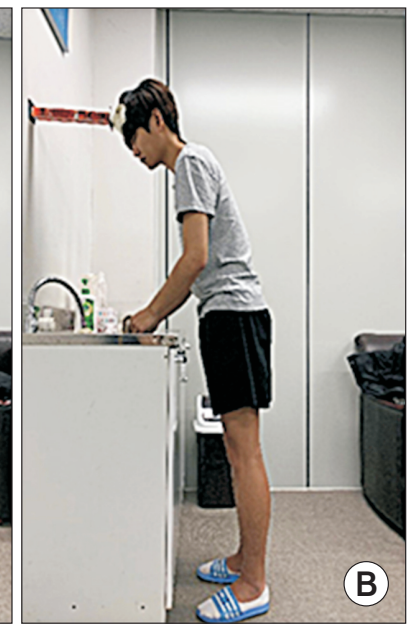

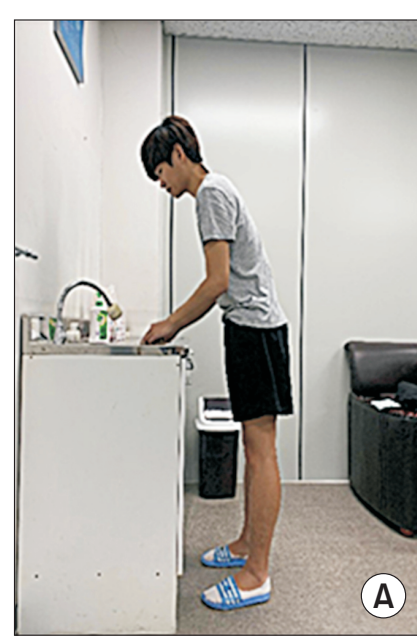

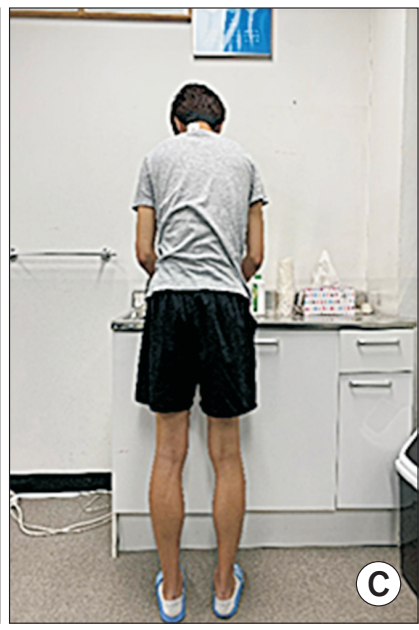

Figure 4. Posture of experiment $(A$, side view with no head support; $B$, side view with head support; C, back view with head support). 
pain in the low back and cervical spine were measured using a 10-point VAS [19].

\section{Statistics}

Statistical analysis of all measurements was performed using SPSS ver. 25.0 for Windows (IBM Co., Armonk, NY, USA). The data did not follow a normal distribution according to the Kolmogorov-Smirnov test. The Wilcoxon signed-rank test was used to compare muscle activity and VAS scores with and without the head support. Statistical significance was set at $\mathrm{p}<0.05$.

\section{RESULTS}

There were significant differences in the activity of the cervical, thoracic, and lumbar erector spinae muscles with and without use of the head support $(p<0.05)$. However, the muscle activity in the gluteus maximus did not differ for the two conditions significantly (Table 2).

There was a significant difference in the VAS scores in the lumbar region with and without the use of the head support ( $p$ $<0.05)$. However, the VAS scores in the cervical region did not significantly differ for the two conditions (Table 3).

\section{DISCUSSION}

In this study, the effects of a head support on the activity of the cervical, thoracic, and lumbar erector spinae were assessed during the task performed in a forward-leaning position. In the forward-leaning posture, the participants demonstrated changes in muscle activity and pain in the cervical and low back when washing dishes using a head support according to sEMG and survey findings. When the head support was used, there were significant differences in the activity of the cervical, thoracic, and lumbar erector spinae but not in the gluteus maximus. When the head support was used, the VAS scores in the low back significantly decreased. There was a trending decrease in the VAS scores in the neck, although these results were not statistically significant.

There were significant decreases in the activity of cervical, thoracic, and lumbar erector spinae with the head support. A previous study that examined the effects of muscle activity with a chest support on a dental stool demonstrated a significant decrease in the sEMG activity of both the thoracic (50\%) and lumbar (33\%) regions during a simulated operating position when compared with a standard dental stool. These findings suggest that the use of a chest support may reduce erector spinae fatigue in dental practitioners [22]. In another study that examined changes in muscle activity with a chest support while operating in a forward-leaning posture, the use of a chest support suggested systematic reduction in muscle activity in the low back and leg muscles [11]. In another previous study, which examined the effects of a passive exoskeleton brace on muscle activity and pain in the lumbar region, the back and leg muscle activity tended to decrease [23]. Specifically, reductions in the thoracic erector spinae activity ranging from $21 \%$ to $31 \%$ and reductions in the lumbar erector spinae activity ranging from $10.3 \%$ to $13.7 \%$ were observed with the use of these devices [23]. However, Parsell et al. [24] showed that a chest support with rigid and mobile arm supports was not effective for reducing low back muscle activity. This find-

Table 2. Comparison of muscle activities of erector spinae and gluteus maximus with and without head support while performing dishwashing

\begin{tabular}{lccrr}
\hline & & \%MVIC & Z-value & \\
\cline { 2 - 3 } Muscle & Head support & No head support & \\
\hline Cervical ES & $5.30 \pm 2.42$ & $10.47 \pm 4.27$ & -4.78 & $<0.001$ \\
Thoracic ES & $15.57 \pm 13.26$ & $22.20 \pm 14.52$ & -3.75 & $<0.001$ \\
Lumbar ES & $12.63 \pm 10.53$ & $16.83 \pm 10.65$ & -3.44 & -1.14 \\
Gmax & $4.78 \pm 3.48$ & $3.48 \pm 5.79$ & 0.001 & 0.254 \\
\hline
\end{tabular}

Values are presented as mean \pm standard deviation. Gmax, gluteus maximus; ES, erector spinae.

Table 3. Comparison of visual analog scale on the cervical and lumbar region with and without head support after dishwashing

\begin{tabular}{|c|c|c|c|c|}
\hline Muscle & Head support & No head support & Z-value & $p$-value \\
\hline Cervical ES & $0.93 \pm 1.38$ & $1.50 \pm 1.35$ & -1.92 & 0.054 \\
\hline Lumbar ES & $0.66 \pm 0.75$ & $2.80 \pm 1.51$ & -4.57 & $<0.001$ \\
\hline
\end{tabular}

Values are presented as mean \pm standard deviation. ES, erector spinae. 
ing implies that the application of inappropriate orthoses overcomes any benefit of a chest support [11]. The muscle activity in the gluteus maximus did not significantly differ with and without use of the head support. The lack of a significant change in muscle activity may have been because the support controlled the lumbar flexion angle at $30^{\circ}$. In conclusion, supporting the body in a forward-leaning posture with a head support systematically reduces muscle activity in the neck and low back muscles.

There was a significant decrease in the VAS scores in the lumbar region with the head support. Although not statistically significant, the VAS scores in the neck area tended to decrease. According to a previous study that examined the effects of a support to decrease the load on muscle activity in the trunk during a forward-leaning posture, the ratio of subjective discomfort significantly decreased (no aid: $<75.2$, aid: 34.0, p < 0.01) [8]. In other previous studies that examined the effects of passive exoskeleton orthoses on lumbar erector spinae activity and discomfort when working in a forward-leaning posture, the discomfort level (approximate perceived discomfort 2/10) in the lumbar region when using the exoskeleton was significantly lower than that without the exoskeleton (approximate perceived discomfort 1/10) [23]. However, one study revealed that if a knee support was not properly used, discomfort was high in both conditions [25]. In another previous study, mechanical neck dysfunction supported with kinesiology tape and postural pump cervical traction, which reduced the muscle effort in the neck, reduced the VAS score and disability index of the neck [26]. Consequently, the use of a head support and chest support when working in a forward-leaning posture is effective in reducing pain by reducing muscle activity in the neck and lumbar region. Therefore, in those who feel pain in the lumbar region and neck while working in a forward-leaning posture, a head support is recommended to reduce pain.

This study has several limitations. First, it is difficult to generalize the results of the study to people of different ages or characteristics than healthy male and female in their 20s. Second, the research was conducted only in a specific posture without consideration of the differences in the methods by which people wash dishes. Third, controlling the angle of the waist at $30^{\circ}$ during the task was difficult. Lastly, A 5-minute break was applied to minimize the effect between measurements, but there was insufficient evidence that it was sufficient time for pain to disappear. To address these limitations, fur- ther research with patients in different age groups, different tasks, and posture control needs to be conducted.

\section{CONCLUSIONS}

The purpose of our study was to determine if the head support was effective in reducing muscle activity and pain in cervical, thoracic, and lumbar erector spinae when working in a forward-leaning posture. There was a significant difference in the muscle activity of the cervical, thoracic, and lumbar erector spinae but not in the gluteus maximus. The VAS scores in the low back significantly decreased, while the VAS scores in the neck did not significantly differ but tended to decrease with the use of the head support. These findings imply that supporting the trunk with a head support decreases the pain that occurs when working in a forward-leaning posture. Therefore, when working in a forward-leaning posture, such as during dishwashing, cooking, and performing the tasks of a factory employee, the use of a head support is recommended.

However, since the head is fixed in the support, it may be poorly functional. In the future, it is necessary to study how the support is devised in a functional manner and at which angle the head should lean to shift the most burden to the waist.

\section{ACKNOWLEDGEMENTS}

We thanks to Na-young Choi, Kwang-hyun Jeong, Ye-jin Shin.

\section{CONFLICTS OF INTEREST}

No potential conflict of interest relevant to this article was reported.

\section{AUTHOR CONTRIBUTIONS}

Conceptualization: TY. Data curation: KK, YK. Formal analysis: KK, YK, TY. Investigation: KK, YK. Methodology: KK, YK, TY. Project administration: KK, YK, TY. Resources: TY. Software: TY. Supervision: TY. Validation: YK. Visualization: KK, TY. Writing - original draft: KK, YK, TY. Writing - review \& editing: TY. 


\section{ORCID}

Kang-hee Kim, https://orcid.org/0000-0001-5610-3402

Yoon-hee Ko, https://orcid.org/0000-0002-5014-485X

\section{REFERENCES}

1. Kim EK, Kim JS. Correlation between rounded shoulder posture, neck disability indices, and degree of forward head posture. J Phys Ther Sci 2016;28(10):2929-32.

2. Lafond D, Champagne A, Descarreaux M, Dubois JD, Prado JM, Duarte M. Postural control during prolonged standing in persons with chronic low back pain. Gait Posture 2009;29(3):421-7.

3. Waters TR, Dick RB. Evidence of health risks associated with prolonged standing at work and intervention effectiveness. Rehabil Nurs 2015;40(3):148-65.

4. Iwakiri K, Kunisue R, Sotoyama M, Udo H. Postural support by a standing aid alleviating subjective discomfort among cooks in a forward-bent posture during food preparation. J Occup Health 2008;50(1):57-62.

5. Tsang SMH, Szeto GPY, Li LMK, Wong DCM, Yip MMP, Lee RYW. The effects of bending speed on the lumbo-pelvic kinematics and movement pattern during forward bending in people with and without low back pain. BMC Musculoskelet Disord 2017;18(1):157.

6. Damecour C, Abdoli-Eramaki M, Ghasempoor A, Neumann WP. Comparison of two heights for forward-placed trunk support with standing work. Appl Ergon 2010;41 (4):536-41.

7. Iwakiri K, Yamauchi S, Yasukouchi A. Effects of a standing aid on loads on low back and legs during dishwashing. Ind Health 2002;40(2):198-206.

8. Iwakiri K, Sotoyama M, Mori I, Saito S. Does leaning posture on the kitchen counter alleviate workload on the low back and legs during dishwashing? Ind Health 2007;45(4):535-45.

9. Yoo WG. Effects of wearing rubber gloves on activities of the forearm and shoulder muscles during different dishwashing stages. J Phys Ther Sci 2015;27(7):2347-8.

10. Albayrak A, van Veelen MA, Prins JF, Snijders CJ, de Ridder H, Kazemier G. A newly designed ergonomic body support for surgeons. Surg Endosc 2007;21(10):1835-40.

11. Albayrak A, Goossens RHM, Snijders CJ, de Ridder H, Kazemier G. Impact of a chest support on lower back muscles activity during forward bending. Appl Bionics Biomech 2010;7(2):
$131-42$

12. Takahashi I, Kikuchi S, Sato K, Sato N. Mechanical load of the lumbar spine during forward bending motion of the trunk-a biomechanical study. Spine (Phila Pa 1976) 2006;31(1):1823.

13. Normand MC, Descarreaux M, Poulin C, Richer N, Mailhot D, Black P, et al. Biomechanical effects of a lumbar support in a mattress. J Can Chiropr Assoc 2005;49(2):96-101.

14. Snijders CJ, Nordin M, Frankel VH. Biomechanica van het spier-skeletstelsel. 5th ed. Springer; 2018;38.

15. Greene J, Wahlund B, Mittlieder J, Lutz A. Electromyographic activity of hamstrings and quadriceps muscle during jumping and landing: pilot study. Grand Forks, North Dakota State University, Doctoral Dissertation. 2018.

16. Gribble PA, Radel S, Armstrong CW. The effects of ankle bracing on the activation of the peroneal muscles during a lateral shuffling movement. Phys Ther Sport 2006;7(1):14-21.

17. Tucker WS, Campbell BM, Swartz EE, Armstrong CW. Electromyography of 3 scapular muscles: a comparative analysis of the cuff link device and a standard push-up. J Athl Train 2008;43(5):464-9.

18. Li H, Cheng W, Liu F, Zhang M, Wang K. The effects on muscle activity and discomfort of varying load carriage with and without an augmentation exoskeleton. Appl Sci 2018;8(12): 2638.

19. Damecour C, Abdoli-Eramaki M, Ghasempoor A, Stevenson J. Comparison of three strategies of trunk support during asymmetric two-handed reach in standing. Appl Ergon 2012;43(1): 121-7.

20. Kõiva R, Hilsenbeck B, Castellini C. Evaluating subsampling strategies for sEMG-based prediction of voluntary muscle contractions. Paper presented at: 2013 IEEE 13th International Conference on Rehabilitation Robotics (ICORR); 2013 Jun 24-26; Seattle, USA. New York: Institute of Electrical and Electronics Engineers, 2013. p. 1-7.

21. Miranda H, Simão R, Moreira LM, de Souza RA, de Souza JA, de Salles BF, et al. Effect of rest interval length on the volume completed during upper body resistance exercise. J Sports Sci Med 2009;8(3):388-92.

22. Tran V, Turner R, MacFadden A, Cornish SM, Esliger D, Komiyama K, et al. A dental stool with chest support reduces lower back muscle activation. Int J Occup Saf Ergon 2016;22(3):301-4.

23. Bosch T, van Eck J, Knitel K, de Looze M. The effects of a pas- 
sive exoskeleton on muscle activity, discomfort and endurance time in forward bending work. Appl Ergon 2016;54: 212-7.

24. Parsell DE, Weber MD, Anderson BC, Cobb GW Jr. Evaluation of ergonomic dental stools through clinical simulation. Gen Dent 2000;48(4):440-4.

25. Iwakiri K, Sotoyama M, Mori I, Jonai H, Saito S. Shape and thickness of cushion in a standing aid to support a forward bending posture: effects on posture, muscle activities and subjective discomfort. Ind Health 2004;42(1):15-23.

26. Dawood RS, Kattabei OM, Nasef SA, Battarjee KA, Abdelraouf OR. Effectiveness of Kinesio Taping versus Cervical Traction on Mechanical Neck Dysfunction. IJTRR 2013;2(2):1. 